\title{
Neuronal Loss in a Lower Motor Neuron Disease Induced by a Murine Retrovirus
}

\author{
Paul Jolicoeur
}

\begin{abstract}
An animal model of neuronal loss induced by a murine retrovirus is described. The neurological disease is manifested by a lower limb paralysis and is characterized as a spongiform myeloencephalopathy. The primary determinant of neurovirulence has been mapped within the viral env sequences and a secondary determinant is present within the LTR region. A model of pathogenesis, suggesting that the disease is receptor-mediated, is proposed.

RÉSUMÉ: Perte neuronale induite par un rétrovirus murin dans une maladie du neurone moteur de la corne antérieure. Nous décrivons un modèle animal de perte neuronale induite par un rétrovirus de la souris. La maladie neurologique se présente comme une paralysie des membres inférieurs et est caractéristique d'une myéloencéphalopathie spongiforme. Le déterminant primaire de la neurovirulence a été cartographié dans la région de l'enveloppe virale et un second déterminant se trouve dans les LTR. On propose un modèle de pathogenèse dans lequel la maladie serait induite par l'intermédiaire d'un récepteur.
\end{abstract}

Can. J. Neurol. Sci. 1991; 18:411-413

The causes of neuronal loss seen in several human neurodegenerative diseases are not known. The etiologic agents are likely to be multiple: They may interact with different cell compartments, through totally distinct mechanisms, or they may possibly affect a common molecular pathway. Loss of neurons may be an active process, resulting from a programmed cell death (apoptosis) or may be passive, as the consequence of inhibition of normal cellular functions. The etiologic agents may be external (toxins, bacteria or viruses) or internal such as a gene defect.

Very few animal models are available to study the mechanisms of neuronal loss adequately. Ideally, such a model should be induced reproducibly in a relatively short time, in an easily available and cheap animal species, with an agent (or a gene product) well characterized and whose fate in vivo is predictable and easy to follow. Historically, viruses have often met many of these criteria because of their simple genetic complexity.

We have been studying a murine retrovirus-induced neurological disease, manifested as a lower motor neuron disease. I will briefly summarize data on this disease. More extensive reviews on this disease have been published. ${ }^{1-6}$

\section{CNS Disease Induced in Mice by Retroviruses (Murine leukemia virus, MuLV).}

The first mouse retrovirus shown to induce a neurological disease was discovered in a population of wild mice in Lake Casitas,California. ${ }^{7,8}$ Some trapped wild mice kept in captivity were found to develop paralysis spontaneously.
Retroviruses isolated from these mice were shown to induce a similar disease in susceptible laboratory mice. One of these isolates, Cas-Br-E MuLV, has been studied in more detail. This virus induces a lower limb paralysis after 2-4 months post-inoculation into newborn mice. Histologically, the disease appears as a spongiform myeloencephalopathy resembling very much the lesions found in scrapie and in human Creutzfeld-Jakob disease or in other human spongiform encephalopathies. However, the lesions are found predominantly in the anterior horn of the lumbar spinal cord, in the brainstem and in the deep cerebellar nuclei. Many regions of the CNS are spared by the disease. The changes involve vacuolization, intense gliosis, neuronal loss and mild demyelination. Typically, no inflammatory cells are seen in degenerative areas.

Essentially, the same disease has also been shown to be induced by laboratory-derived variants (temperature-sensitive mutants) of Moloney MuLV. ${ }^{9}$

\section{Genetic Elements of the Viral Genome Determining the Neurovirulence}

Molecular cloning of the wild type Cas-Br-E MuLV10 or of a Moloney MuLV variant ${ }^{11}$ established that these retroviruses were responsible for the disease. The availability of these cloned DNA genomes allowed the construction of chimeric viral genomes by recombinant DNA technology. These chimeric viral genomes, made with parental DNA from the neurotropic MuLV and a non-paralytogenic MuLV, have allowed the mapping of

From the Laboratory of Molecular Biology, Clinical Research Institute of Montreal, Montreal

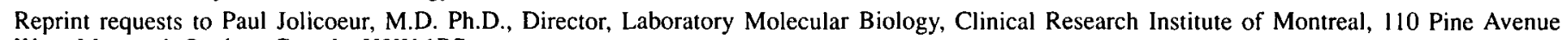
West, Montreal, Quebec, Canada H2W 1 R7 
the sequences responsible for inducing the spongiform degeneration, both in Cas-Br-E MuLV12,13 and in ts-1 Moloney MuLV, ${ }^{14}$ within the env sequences encoding the gp 70 protein. A murine retrovirus harbors only 3 genes encoding proteins: the gag (encoding structural proteins), the pol (encoding the reverse transcriptase and the integrase) and the env (encoding the proteins of the envelope). These genes are flanked by two direct repeat sequences, the long terminal repeats (LTR). The env gp70 protein is the protein responsible for the recognition of the cellular receptor to initiate infection.

In addition, using the same strategy of constructing chimeric MuLVs, we have been able to identify an additional determinant of neurovirulence within the LTR region of the genome. By exchanging these sequences, we were able to produce chimeric MuLVs which induced disease with a different clinical presentation. ${ }^{15,16}$ We found that these viruses induced the same spongiform lesions as the wild-type virus, but either in novel CNS areas or/and more extensively in some regions than those affected by the wild-type Cas-Br-E MuLV. This result indicates that the LTR region determines the severity of the lesions, most likely through controlling the rate of virus replication. These LTR sequences appear to determine the distribution of the lesions within the CNS, most likely through a preferential replication of the virus in some CNS cells harboring the necessary transcription factors for a given LTR.

\section{The Neuronal Loss in Cas-Br-E MuLV-Induced Disease is Indirect}

To study the mechanism by which Cas-Br-E MuLV induces disease, we determined in which cells it was replicating within the CNS. We used the in situ hybridization technique with a probe specific for the inoculated virus (Cas-Br-E MuLV) and which did not detect endogenous viral sequences. ${ }^{17}$ The specificity of this probe must be emphasized, since the normal mouse genome contains several copies of endogenous MuLV sequences which can be induced by various factors. It was therefore important to avoid the detection of these endogenous viral sequences in our study.

We found that the number of infected cells detected in areas showing spongiform degeneration correlated with the severity of the pathological lesions. ${ }^{15,18}$ However, this correlation did not hold for all CNS regions, since infected cells were also found in regions which never show signs of pathology, indicating that these regions are resistant to the detrimental effect of this virus. ${ }^{18}$ This result also indicated that the distribution of infected cells within the CNS is not at random, but appears to reflect an important characteristic of the virus-cell interactions. Interestingly, all infected cells in regions with spongiform changes appeared morphologically as non-neuronal cells (glial and endothelial cells), suggesting that the neuronal loss seen in this disease is indirect and is not the consequence of infection of the neurons. 18 When young animals (10-day old) inoculated at birth were examined, a greater population of the infected cells were identified as endothelial cells, suggesting that the virus gets into the CNS through infection of endothelial cells and not through penetration into the CNS of already infected cells. ${ }^{18}$ The distribution of the infected endothelial cells in these young mice reflected the distribution of the lesions seen later in dis- eased mice, indicating that the distribution of the lesions is laid out early and may reflect in part the site of the initial infection.

Since endothelial and glial cells are not infected at random in this disease, our results suggest that some cell factors (possibly transcription factors) are distributed differently in these nonneuronal cells and affect differently the replication of Cas-Br-E MuLV. Consequently, our data suggest that CNS non-neuronal cells are not all the same, and are different in various regions of the CNS. 18

Our findings contrast significantly with those of Sharpe et al. who reported abortive replication of Cas-Br-E MuLV within neurons of diseased mice. 19 The use of non-specific antibodies able to detect several classes of exogenous and endogenous viral proteins may have allowed the detection of endogenous viral proteins in their study.

\section{A Model of Neuronal Loss Induced by Murine Retrovirus}

With the available knowledge on this retrovirus-induced disease, we have proposed a model to explain its pathogenesis. $1,4,10,13,18$ Since the env sequences encoding the gp 70 protein harbor the determinant of spongiform degeneration and since retroviral env proteins are known to recognize receptors, we hypothesized that the disease is receptor-mediated. This putative receptor may be present on neurons. The gp70 proteins (free or virion-associated) could compete with a trophic growth factor acting through this receptor and which is essential for neuron survival. Alternatively, binding of gp 70 proteins to this receptor could itself induce cell death, either by apoptosis or by other mechanisms such as inappropriate opening of channels. This putative receptor may also be localized on non-neuronal cells. Its occupancy by gp 70 proteins may lead to a higher production, by these non-neuronal cells, of a factor toxic for neurons or to a decreased production, by these non-neuronal cells, of a factor essential for neuron survival.

This model contrasts with another model in which abnormal processing of gp70 precursor would lead to cell degeneration.6,20 This latter model supposes that neurons are infected, an observation that we have been unable to substantiate. ${ }^{18}$

\section{How Relevant is this Retrovirus-Induced Disease as a Model for Other Human Neurodegenerative Diseases?}

The present model may be important in understanding the human neurological diseases induced by retroviruses, namely by HIV and HTLV-I. The AIDS dementia complex, which is apparently induced by HIV, does not seem to be induced through a direct infection of neurons by HIV, but is thought to result from an indirect effect of HIV on neurons. ${ }^{21}$ This mode of pathogenesis is similar to what we are proposing for this mouse disease. This murine model may therefore be quite instrumental in understanding the HIV-induced human CNS disease. The mechanism by which HTLV-I induces the tropical spastic paraparesis in a small percentage of infected individuals is totally unknown. ${ }^{22}$ This mouse model of retrovirus-induced CNS disease may also help to probe the exact nature of this human disease, although the types of CNS lesions are distinct in both diseases.

Interestingly, the spongiform lesions seen in this murine model are very similar to those found in scrapie and CreutzfeldJakob disease. These latter diseases are almost certainly not induced by a retrovirus, but appear to be caused by an unusual 
agent, possibly the prion itself. ${ }^{23}$ Although the etiologies of these human and mouse diseases are apparently quite different, the fact that they exhibit the same type of lesions (a form of neurodegeneration which is rare and quite specific) suggests that they may share a common molecular pathway. A better understanding of this murine retrovirus-induced disease may therefore lead to a better knowledge of the pathogenesis of CreutzfeldJakob disease.

In this retrovirus-induced murine disease, the distribution of the lesions and the neurons affected by the virus are the same as those in amyotrophic lateral sclerosis. ${ }^{24}$ These neurons may degenerate through the same, or through a distinct, mechanism. However, it is likely that these neurons exhibit some characteristics which set them apart from the others. It is not unrealistic to predict that the information obtained on this murine disease will be quite relevant to amyotrophic lateral sclerosis.

In addition, several neurodegenerative diseases are characterized by loss of neurons. Most likely, several molecular mechanisms of cell death are operating in these various diseases. Although most of them are obviously not induced by a retrovirus, it is not unlikely that the molecular mechanism of cell death, seen in this murine retrovirus-induced disease, may be shared with one or many of these human diseases.

\section{ACKNOWLEDGEMENTS}

Work in our laboratory was supported by grants from the Medical Research Council of Canada and from the Amyotrophic Lateral Sclerosis Association (USA). I thank my colleagues Claude Gravel, Denis D. Kay and Yves Robitaille for stimulating discussions.

\section{REFERENCES}

1. Jolicoeur P. Retrovirus-induced lower motor neuron disease in mice: A model for amyotrophic lateral sclerosis and human spongiform neurological diseases. In: Hudson AJ: Amyotrophic Lateral Sclerosis. Concepts in Pathogenesis and Etiology, Toronto: University of Toronto Press 1990: 53-82.

2. Gardner MB. Type-C viruses of wild mice: characterization and natural history of amphotropic, ecotropic and xenotropic murine leukemia viruses. Curr Top Microbiol Immunol 1978; 79: 215239.

3. Garnder MB. Retroviral spongiform polioencephalomyelopathy. Rev Infect Dis 1985; 7: 99-110.

4. Jolicoeur P, Rassart E, DesGroseillers L, et al. Retrovirus-induced motor neuron disease of mice: molecular basis of neurotropism and paralysis. In: Rowland, Lewis P: Amyotrophic Lateral Sclerosis: Raven Press Limited 1991: 481-493.

5. Zachary JF, Knupp CJ, Wong PKY. Noninflammatory spongiform polioencephalopathy caused by a neurotropic temperature -sensitive mutant of Moloney murine leukemia virus TB. Am J Pathol 1986; 124: 457-468.

6. Wong PKY. Moloney murine leukemia virus temperature-sensitive mutants: a model for retrovirus-induced neurological disorders. Curr Top Microbiol Immunol 1990; 160: 29-60.

7. Officer JE, Tecson N, Estes JD, et al. Isolation of a neurotropic type-C virus. Science 1973; 181: 945-947.

8. Gardner MB, Henderson BE, Officer JE, et al. A spontaneous lower motor neuron disease apparently caused by indigenous type-C RNA virus in wild mice. J Natl Cancer Inst 1973; 51: 1243-1254.
9. McCarter JA, Ball JK, Frei JV. Lower limb paralysis induced in mice by a temperature-sensitive mutant of Moloney leukemia virus. J Natl Cancer Inst 1977; 59: 179-183.

10. Jolicoeur P, Nicolaiew N, DesGroseillers L, et al. Molecular cloning of infectious viral DNA from ecotropic neurotropic wild mouse retrovirus. J Virol 1983; 45: 1159-1163.

11. Yuen PH, Malehorn D, Nau C, et al. Molecular cloning of two paralytogenic, temperature-sensitive mutants, ts 1 and $t s 7$, and the parental wild-type Moloney murine leukemia virus. J Virol 1985; 54: 178-185.

12. DesGroseillers L, Barrette M, Jolicoeur P. Physical mapping of the paralysis-inducing determinant of a wild muse ecotropic neurotropic retrovirus. J Virol 1984; 52: 356-363.

13. Paquette $Y$, Hanna $Z$, Savard $P$, et al. Retrovirus-induced murine motor neuron disease: Mapping the determinant of spongiform degeneration within the envelope gene. Proc Natl Acad Sci USA 1989: 86: 3896-3900.

14. Szurek PF, Yuen PH, Ball JK, et al. A val-25-to ile substitution in the envelope precursor polyprotein, gPr80env, is responsible for the temperature sensitivity, inefficient processing of gPr80env, and neurovirulence of tsl, a mutant of Moloney murine leukemia virus TB. J Virol 1990; 64: 467-475.

15. Paquette Y, Kay DG, Rassart E, et al. Substitution of the U3 long terminal repeat region of the neurotropic $\mathrm{Cas}-\mathrm{Br}-\mathrm{E}$ retrovirus affects its disease-inducing potential. J Virol 1990; 64: 37423752.

16. DesGroseillers L, Rassart E, Robitaille Y, et al. Retrovirus-induced spongiform encephalopathy: The 3 '-end long terminal repeatcontaining viral sequences influence the incidence of the disease and the specificity of the neurological syndrome. Proc Natl Acad Sci USA 1985; 82: 8818-8822.

17. Rassart E, Nelbach L, Jolicoeur P. Cas-Br-E murine leukemia virus: Sequencing of the paralytogenic region of its genome and derivation of specific probes to study its origin and the structure of its recombinant genomes in leukemic tissues. J Virol 1986; 60: $910-919$

18. Kay D, Gravel C, Robitaille Y, et al. Retrovirus-induced spongiform myeloencephalopathy in mice: Regional distribution of infected target cells and neuronal loss occurring in the absence of viral expression in neurons. Proc Natl Acad Sci USA 1991; 88: 1281-1285.

19. Sharpe AH, Hunter JJ, Chassler P, et al. Role of abortive retroviral infection of neurons in spongiform CNS degeneration. Nature 1990; 346: 181-183.

20. Yuen PH, Tzeng E, Knupp C, et al. The neurovirulent determinants of ts I, a paralytogenic mutant of Moloney murine leukemia virus TB, are localized in at least two functionally distinct regions of this genome. J Virol 1986; 59: 59-65.

21. Price RW, Brew B, Sibtis J, et al. The brain in AIDS: Central nervous system HIV infection and AIDS dementia complex. Science 1988; 239: 586-592.

22. McFarlin DE, Koprowski H. Neurological disorders associated with HTLV-I. Curr Top Microbiol Immunol 1990; 160: 99-119.

23. Prusiner SB. Prions and neurodegenerative diseases. N Engl J Med 1987; 317: 1571-1581.

24. Gardner MB, Rasheed S, Klement V, et al. Lower motor neuron disease in wild mice caused by indigenous type $C$ virus and search for a similar etiology in human amyotrophic lateral sclerosis. In: Andrews JM, Johnson, RT, Brazier MAB: Amyotrophic Lateral Sclerosis. New York: Academic Press 1976; $217-$ 234. 